\title{
Grammaticalisation, métonymie et pertinence
}

\author{
Walter De Mulder \\ Université d'Anvers
}

\section{Introduction}

Les changements sémantiques sous-jacents aux phénomènes de grammaticalisation ont été analysés dans le passé comme des transferts métaphoriques, des glissements métonymiques ou des « conventionnalisations d'implicatures ». ${ }^{1}$ Dans cette brève contribution, nous nous demanderons, à l'instar de Nicolle (1998), quel pourrait être l'apport de la théorie de la pertinence à l'analyse de ces évolutions sémantiques. Puisque Nicolle (1998) s'est servi, entre autres, de l'évolution de la construction anglaise be going to + infinitif pour illustrer ses idées, nous prendrons comme point de départ de cette étude l'analyse de la grammaticalisation de la séquence aller + infinitif proposée par Detges (1999). Nous expliquerons d'abord pourquoi il est nécessaire de combiner son analyse métonymique avec les mécanismes pragmatiques décrits par la théorie de la pertinence. Ensuite, nous montrerons brièvement qu'en plus des éléments déjà mentionnés, il faut aussi tenir compte de la fréquence d'emploi de la séquence aller + infinitif.

\section{L'évolution de la séquence aller+ infinitif}

La grammaticalisation de la séquence aller + infinitif a été décrite par plusieurs auteurs ; nous partirons ici de l'analyse proposée par Detges (1999), que nous compléterons toutefois en nous servant d'idées et de données empruntées à Gougenheim (1929), Flydal (1943), Wilmet (1970) et Werner (1980). Selon Detges (1999), le mécanisme sous-jacent au changement sémantique du verbe aller, qui s'est transformé de verbe de mouvement en marqueur du «futur périphrastique » (du moins lorsqu'il est suivi d'un infinitif), est de nature métonymique, mais la cause et la direction du processus de grammaticalisation (du concret vers l'abstrait) doivent être expliquées en faisant appel à des stratégies et à des intentions communicatives particulières des locuteurs.

A l'instar de Blank (1997), Detges (1999: 36) considère comme métonymiques des changements sémantiques du type 'contenant $>$ contenu', 'acte > résultat', etc. A son avis, ces glissements de sens s'expliquent à partir des relations (non linguistiques) existant entre les concepts qui représentent les entités désignées par les signes linguistiques. Ces concepts sont organisés en cadres (frames), c'est-à-dire des ensembles de connaissances encyclopédiques. Ainsi, en ce qui concerne le concept tête, nous savons (i) que la tête est une partie du corps et, par extension, de la personne humaine, (ii) que c'est la partie supérieure du corps (du moins chez les êtres humains), (iii) que la tête est le siège de l'intellect, etc. Lorsque le contexte met au premier plan certaines connaissances qui font partie du cadre associé au nom tête, et en repousse d'autres à l'arrière-plan, ce nom acquiert différentes interprétations. Il peut ainsi désigner, selon le Petit Robert, les personnes humaines (ex. 1) ou l'intellect dont la tête est censée être le siège (ex. 2) :

(1) Une tête couronnée (Petit Robert, s.v. tête)

(2) On n'écrit pas avec son cœur, mais avec sa tête (Flaubert, cité par le Petit Robert, s.v. tête) 
Dans l'exemple (1), c'est l'idée de la personne humaine, dont la tête est une partie, qui a été mise au premier plan, dans l'exemple (2), par un mouvement en quelque sorte inverse, c'est l'intellect (qui est censé être «dans» la tête) qui se voit promu au premier plan. Mais dans les deux cas, la métonymie résulte de glissements à l'intérieur du cadre associé à tête (Koch 1996, 1999) : tête > personne ; tête > intellect. De plus, elle consiste à changer les rapports premier plan / arrière-plan existant au sein du cadre.

Lorsqu'il est question de verbes, les cadres qu'ils désignent se reflètent dans leur valence (Detges 1999 : 37) ; aller désigne ainsi un cadre qui comporte (i) deux éléments centraux ou actants, à savoir (a) l'agent du mouvement et (b) la destination de ce mouvement, mais aussi (ii) plusieurs circonstants, comme (a) la vitesse, (b) l'instrument et (c) l'intention avec laquelle l'agent se dirige vers sa destination. Selon Detges, la grammaticalisation liée au verbe aller est le résultat de glissements métonymiques à l'intérieur de ce cadre. Il distingue deux étapes :

2.1. du mouvement à l'intention ;

2.2. de l'intention au futur.

Nous présentons ci-dessous son analyse des glissements métonymiques impliqués.

\subsection{Du mouvement à l'intention}

L'intention associée au verbe aller concerne souvent les actes que l'auteur du mouvement se propose d'exécuter lorsqu'il arrive à la destination du mouvement:

(3) Car incontinant le roy manda tous ses barons, cappitaines et chefz de guerre, et sans aulcun delay fit appareillier tout ce qui estoit de besoing pour aller en Espaigne commancer la guerre contre les barons du pays. (Jehan de Paris 8, cité par Werner 1980: 131)

Il suffit que cette intention soit plus importante en contexte que la destination à laquelle elle est associée pour que seule l'intention soit exprimée. Detges (1999: 39) cite ainsi les exemples suivants, dans lesquels seule l'intention est encore explicitée,

- soit parce que le (co-)texte précise la destination du mouvement:

(4) Nos alomes la messe oï; Tuit alomes vers le mostier. (Roman de Renart 12582, fin $12^{\mathrm{e}}-$ début $13^{\mathrm{e}}$ s.; cité par Littré 1961/62 et Detges 1999: 39)

- soit parce que la destination peut être déduite à partir de nos connaissances encyclopédiques («toute action a lieu à un endroit particulier»):

(5) Il meismes ala trois serjans apeler (Li romans de Berte aus grans pies XVII, fin $13^{\mathrm{e}}$ siècle, cité par Littré 1961/62 et Detges 1999: 39)

Bref, il n'est pas toujours nécessaire d'expliciter la destination parce que celle-ci peut être déduite sans problèmes du contexte. Partant, le verbe aller s'emploiera par la suite dans des contextes où il n'exprime plus l'idée d'un mouvement, mais où il signale seulement la présence d'une intention:

(4) Il est bien temps de deviser / Les personnaiges et nommer. / Je vous les veulx nommer à tous. / Je voys au Monde commencer. (Moralité de Charité, 1532-1550, passage cité par Gougenheim 1929/1971: 98 et Detges 1999: 39)

Comme le fait remarquer Detges (1999: 40), en (4) je voys n'exprime plus le mouvement, puisque le locuteur ne doit pas se déplacer pour présenter les personnages. Je voys correspond plutôt à «j'ai l'intention (de) ... », ce qui est d'ailleurs étayé par la juxtaposition de je voys avec je veulx. Le résultat est que l'intention est mise au premier plan, alors que la destination est repoussée à l'arrière-plan.

L'analyse n'est pas encore complète, toutefois : il faut encore expliquer ce qui déclenche le changement sémantique. Detges (1999 : 48) avance à ce sujet que les locuteurs font appel à des verbes désignant un mouvement visible pour exprimer leurs intentions ou pour faire des assertions sur le futur, qui n'est pas 
perceptible, afin de signaler à leurs interlocuteurs que ces intentions ou ces événements futurs vont effectivement être réalisés. Il est intéressant de noter à ce propos que le changement sémantique du verbe aller décrit dans cette partie de notre texte se produit initialement dans des contextes où ce verbe est conjugué à la première personne (Gougenheim 1929, Flydal 1943, Wilmet 1970 et Werner 1980). Cela s'explique en effet, selon Detges (1999: 40), par le fait que la périphrase aller + infinitif y sert surtout à exprimer des actes de langage comme la promesse, des actes dans lesquels l'intention de faire quelque chose occupe le premier plan. En disant «je vais ...», le locuteur signale qu'au moment de parler, il a déjà entamé le mouvement qui conduira à la réalisation de l'acte (voir également Flydal 1943: 8). Le rapport métonymique entre mouvement et intention est ainsi ancré dans l'expérience du monde du locuteur et de son interlocuteur, ce qui contribue à rendre crédible pour l'interlocuteur l'idée que l'acte visé va se réaliser ; la métaphore, qui est basée sur un rapport conceptuel créé par le locuteur, n'aurait pas le même effet.

Le changement de sens décrit implique donc deux niveaux : (i) un niveau cognitif, où l'on trouve les rapports conceptuels (konzeptuelle Brücken, Blank 1997 : 295) sous-jacents à l'évolution sémantique, et (ii) un niveau pragmatique, qui comporte le besoin communicatif, ressenti par le locuteur, de rendre crédible l'idée que ses actes intersubjectifs, comme la promesse, vont se réaliser et qui explique ainsi (i) le recours initial au concept concret d'aller et, du coup, (ii) la direction du changement (du concret vers l'abstrait) (Detges 1999 : 41).

Si l'analyse de Detges nous semble tout à fait convaincante, l'on peut y ajouter un élément, qui pourrait fournir une réponse supplémentaire à la question posée ci-dessus, concernant l'élément qui déclenche le processus de grammaticalisation. Selon Koch (2004 : 12), en effet, l'élément déclencheur des glissements métonymiques serait fourni par le principe de pertinence. Rappelons que selon ce principe, nos énoncés créent des attentes de pertinence optimale :

(...) le destinataire d'un énoncé est en droit d'attendre, d'une part, que cet énoncé soit au moins assez pertinent pour valoir la peine d'être traité (...) et d'autre part, qu'il soit l'énoncé le plus pertinent compte tenu des capacités et des préférences du locuteur. (Wilson $2006: 42$ )

La pertinence est définie en termes d'efforts de traitement et d'effets cognitifs (comme les implications contextuelles vraies, les renforcements ou les révisions d'hypothèses existantes, etc.) :

Toutes choses étant égales par ailleurs, plus un stimulus produit d'effets cognitifs, plus il sera pertinent pour l'individu qui l'a traité ;

(...) toutes choses étant égales par ailleurs, moins un énoncé demande d'effort de traitement, plus il est pertinent. (Wilson $2006: 44$ )

Notons que ces principes permettent entre autres d'expliquer pourquoi le locuteur n'exprime plus la destination dans les exemples (4) et (5): puisque cette information peut être déduite du contexte, le locuteur rend son énoncé plus pertinent s'il ne la reprend pas, réduisant ainsi l'effort de traitement pour son interlocuteur. La présence des éléments contextuels décrits par Detges (la première personne, etc.) permet en outre à l'interlocuteur d'identifier à peu de « frais » de traitement l'intention communicative du locuteur. La combinaison du sens avec les éléments contextuels crée donc des effets contextuels supplémentaires. Ces analyses doivent peut-être encore être affinées, mais elles confirment à notre avis l'hypothèse que la recherche de la pertinence optimale pourrait expliquer pourquoi s'opèrent les glissements métonymiques décrits par Detges.

\subsection{2. De l'intention au futur}

Après le premier glissement métonymique, la séquence aller + infinitif n'est pas encore transformée en marqueur grammatical ; la généralisation qui en résulte et qu'on retrouve dans l'évolution de la structure be going to + infinitif en anglais, concerne selon Nicolle (1998) deux sens lexicaux. Le deuxième glissement métonymique, qui transforme la séquence aller + infinitif en marqueur grammatical, est basé sur l'idée généralement acceptée selon laquelle la réalisation de nos intentions est normalement située dans le futur : si les actes désignés par l'infinitif après le verbe aller renvoient aux intentions du sujet du 
verbe et que le locuteur essaie en outre de rendre crédible l'idée que ces intentions vont être réalisées, on comprend que la séquence aller + infinitif puisse s'employer par la suite pour exprimer l'idée de futur. Cette transition peut s'observer dans des passages comme (7), où aller + infinitif exprime selon Detges (1999: 43) soit l'intention, soit le futur:

$$
\begin{aligned}
& \text { Je lui voys mander un cartel (Rabelais, Pantagruel IV, 32, 1532, cité par Gougenheim 1929: } 99 \\
& \text { et Detges 1999: 43) }
\end{aligned}
$$

L'interprétation finale de cet énoncé dépend de ce qu'on met à l'avant-plan : l'intention ou le futur ; on assiste donc de nouveau à un glissement métonymique. Le développement qui permettra finalement à aller + infinitif de n'exprimer que l'idée de futur est accompagné d'un mouvement de généralisation, lors duquel le verbe commence à être employé avec des infinitifs désignant des événements qui ne dépendent plus de l'intention du sujet. Cette évolution est illustrée par les exemples suivants :

(8) Par deffaulte de patience, / Tu vas perdre ta conscience. (Moralité de Charité, 1532-1550, cité par Gougenheim 1929 / 1971: 98 et Detges 1999: 42)

(9) La paix va refleurir, les beaux jours vont renaître. (Racine, Andromaque, II, 4, 1667, cité par Littré 1961/62 et Detges 1999: 42).

La valeur future de aller + infinitif se distingue toujours de celle du futur simple, puisque l'indicatif présent du verbe aller + infinitif rattache le procès désigné au présent : le futur est appréhendé à partir du présent, «comme le développement naturel de l'actualité » (Wilmet 1970: 195). La motivation pragmatique décrite par Detges reste donc présente, même lorsque le sujet du verbe ne renvoie plus au locuteur. Ainsi Leeman-Bouix (1994 : 163) note que, même si le futur périphrastique ne correspond pas à un procès immédiat, comme dans Max et Léa vont se marier, il présente ce procès comme tel "pour en garantir la réalisation ", à l'opposé du futur simple.

L'article de Nicolle (1998) permet d'analyser aussi cette deuxième étape de l'évolution de aller + infinitif en des termes empruntés à la théorie de la pertinence. Nicolle note d'abord que si la grammaticalisation est définie comme un processus qui change un élément lexical en un élément grammatical, elle peut être définie dans le cadre de la théorie de la pertinence comme une évolution qui change des termes exprimant des informations conceptuelles en des termes exprimant des informations procédurales (voir Traugott et Dasher (2002 : 10, 40) pour des idées comparables). En effet, dans la théorie de la pertinence, l'information conceptuelle permet d'élaborer une représentation de la scène décrite par l'énoncé, alors que l'information procédurale donne des instructions sur la façon dont il faut organiser et manipuler cette information conceptuelle ; le premier type d'information correspond donc (en gros) au sens lexical et le second au sens grammatical. Cette distinction permet à Nicolle (1998: 10) d'expliquer pourquoi une expression qui (comme aller + infinitif) a développé un sens procédural en plus de son sens conceptuel, se transforme en marqueur grammatical. Lorsqu'un interlocuteur doit interpréter un énoncé comportant une telle forme (comme aller + infinitif) dans un énoncé (comme (7)), où des éléments contextuels favorisent l'activation du nouveau sens procédural (dans le cas de aller + infinitif, le sens temporel), cette forme pourra recevoir deux interprétations, puisque son sens lexical - ou conceptuel - sera également activé. ${ }^{2}$ Or le nouveau sens procédural apporte des informations sur la façon dont l'énoncé doit être interprété et contribue ainsi à réduire l'effort de traitement - le sens temporel permet, en gros, de situer la situation dénotée sur l'axe du temps. Si l'activation du sens procédural permet à l'interlocuteur de récupérer suffisamment d'effets contextuels, le processus interprétatif s'arrêtera, conformément au principe de pertinence, et seul le sens procédural sera retenu - donc, en ce qui concerne aller + infinitif, le sens temporel. En effet, le traitement supplémentaire de l'information conceptuelle (uniquement celle exprimée par la structure aller + infinitif dans notre cas) implique un effort supplémentaire qui ne sera pas récompensé par des effets cognitifs supplémentaires (Nicolle 1998 : 10). 


\section{Pertinence, métonymie et ... fréquence}

Les analyses qui viennent d'être présentées, gagneraient certainement à être précisées davantage et à être illustrées par d'autres exemples. Il faudrait encore expliquer, entre autres,

- $\quad$ pourquoi l'emploi d'aller + infinitif pour exprimer (l'aspect inchoatif dans) le passé en moyen français n'est plus très fréquent en français moderne ;

- $\quad$ pourquoi aller + infinitif a été retenu comme forme du futur périphrastique, alors que devoir + infinitif et vouloir + infinitif ont disparu (à quelques rares exceptions près).

Cela permettrait entre autres de décrire de façon plus détaillée le rôle du contexte dans l'évolution de la séquence aller + infinitif. Nous devrons aussi justifier de façon plus explicite pourquoi nous considérons que c'est l'ensemble de la séquence et pas le verbe aller seul qui est grammaticalisé et qui exprime le futur périphrastique. Cette hypothèse semble être étayée par le fait que la séquence ne s'emploie qu'au présent et à l'imparfait, ce qui n'est évidemment pas le cas pour le verbe aller seul. En outre, les inférences décrites par Detges prennent comme point de départ l'ensemble de la séquence (y compris la personne grammaticale) et non le verbe aller seul.

Nous croyons toutefois que ces précisions ne remettraient pas en cause l'intervention du principe de pertinence comme élément déclencheur du processus de grammaticalisation. On notera d'ailleurs à ce propos que le fait même que la séquence aller + infinitif se transforme en marqueur de temps contribue à augmenter la pertinence de l'énoncé, dans la mesure où les informations procédurales qu'elle exprime réduisent le coût de traitement.

Précisons toutefois que, de toute évidence, la théorie de la pertinence ne suffit pas à elle seule pour décrire les changements observés : ainsi que l'ont noté Ruiz de Mendoza Ibáñez et Hernández (2003 : 29), ${ }^{3}$ la recherche de la pertinence optimale déclenche les changements sémantiques, elle explique pourquoi les interlocuteurs préfèrent certaines interprétations des énoncés produits à d'autres, mais elle n'explique pas, par exemple, pourquoi on passe de l'expression de la destination à celle de l'intention ; pour cela, on a besoin de représentations cognitives comme les cadres décrits par Detges (1999), leurs composantes et les relations (associatives et autres) qui existent entre ces composantes et qui rendent possibles les glissements métonymiques (voir également Koch 2004).

Enfin, il faut noter, avec Koch (2004), que la théorie de la pertinence permet d'analyser la phase initiale du changement de sens, lors de laquelle les formes sont interprétées d'une façon nouvelle, mais que l'évolution sémantique n'est vraiment complète que si l'innovation sémantique entre dans la langue, c'est-à-dire si elle fait partie du sens conventionnel des termes linguistiques. Or il n'est pas évident de décrire cette conventionnalisation dans le cadre de la théorie de la pertinence, puisque celle-ci considère que toutes les implicatures sont liées au contexte et qu'elle tend à rejeter pour cette raison l'idée qu'il existe des implicatures conversationnelles généralisées. Cependant, si le glissement métonymique que déclenche la recherche de la pertinence optimale consiste à mettre le sens temporel au premier plan et à repousser le sens intentionnel à l'arrière-plan, il ne serait pas surprenant que ce sens temporel plus saillant soit finalement associé comme sens codé à la séquence aller + infinitif, plutôt que le sens intentionnel moins saillant. Il faut toutefois, pour que le nouveau sens soit associé à la séquence, que l'inférence du sens intentionnel au sens temporel se répète fréquemment (Traugott et Dasher 2002). Il ne faut donc pas oublier le rôle essentiel joué par la fréquence dans la conventionnalisation, même si le résultat final de l'évolution satisfait au principe de pertinence (en effet, lorsque l'information procédurale temporelle est conventionnalisée, elle est plus accessible à l'interlocuteur que si celui-ci doit l'identifier par des inférences). Cette observation soulève la question de savoir si la théorie de la pertinence est compatible avec toutes les conséquences d'une approche de la grammaticalisation basée sur la notion de fréquence telle que décrite, entre autres, par Bybee (2006). Partant, ce texte ne saurait être qu'un point de départ pour des recherches futures, lors desquelles nous aimerions également reprendre les questions formulées au début de ce paragraphe. A suivre, donc. 


\section{Références}

Blank, Andreas (1997). Prinzipien des lexikalischen Bedeutungswandel am Beispiel der romanischen Sprachen. Tübingen: Niemeyer.

Bybee, Joan L., Perkins, R.D., et Pagliuca, W. (1994). The Evolution of Grammar : Tense, Aspect and Modality in the Languages of the World. Chicago : Chicago University Press.

Bybee, Joan (2006). «From usage to grammar: The mind's response to repetition ». Language 82/4, 711-733.

Detges, Ulrich (1999). «Wie entsteht Grammatik? Kognitive und pragmatische Determinanten der Grammatikalisierung von Tempus Markern. » In Lang, Jürgen, et Neumann-Holzschuh, Ingrid, éds. Reanalyse und Grammatikalisierung in den romanischen Sprachen. Tübingen : Max Niemeyer Verlag, 31-52.

Flydal, Leiv (1943). Aller et venir de suivis de l'infinitif comme expressions de rapports temporels. Oslo: I Kommisjon Hos Jacob Dybwad.

Fries, Charles C. (1927). « The expression of the future. » Language 3, 87-95.

Gougenheim, Georges $(1929$ : 1971). Etude sur les périphrases verbales de la langue française. Paris : Nizet.

Hopper, Paul, et Traugott, Elizabeth (2003). Grammaticalization. Cambridge: Cambridge University Press. Deuxième édition.

Koch, Peter (1996). «Der Beitrag der Prototypetheorie zur Historischen Semantik: Eine kritiche Bestandsaufnahme ». Romanistisches Jahrbuch 46, 27-46.

Koch, Peter (1999). «Frame and contiguity. On the cognitive bases of metonymy and certain types of word formation ». In Panther, Klaus-Uwe, et Radden, Günter, éds. Metonymy in Thought and Language. Amsterdam / Philadelphia : John Benjamins, 139-167.

Koch, Peter (2004). « Metonymy between pragmatics, reference, and diachrony ». Metaphorik.de 07/2004.

Kuteva, Tania (2001). Auxiliation. An enquiry into the nature of grammaticalization. Oxford : Oxford University Press.

Leeman-Bouix, Danielle (1994). Grammaire du verbe français. Des formes au sens. Paris : Nathan.

Littré, Emile (1961/62). Dictionnaire de la langue française. Paris: Gallimard.

Nicolle, Steve (1998). «A relevance theory perspective on grammaticalization ». Cognitive Linguistics 9-1, 1-35.

Ruiz de Mendoza Ibáñez, Francisco José, et Hernández, Lorena Pérez (2003). « Cognitive operations and pragmatic implication ». In Panther, Klaus-Uwe, et Thornburg, Linda L., éds. Metonymy and Pragmatic Inferencing. Amsterdam / Philadelphia : John Benjamins, 23-49.

Sperber, Dan et Wilson, Deirdre (1995). Relevance: Communication and Cognition. Oxford: Basil Blackwell. Deuxième édition.

Traugott, Elizabeth C. \& Dasher, Richard B. (2002). Regularity in Semantic Change. Cambridge: Cambridge University Press.

Werner, Edeltraut (1980). Die Verbalperiphrase im Mittelfranzösischen. Eine semantisch-syntaktische Analyse. Frankfurt a.M. : Lang.

Wilmet, Marc (1970). Le système de l'indicatif en moyen français. Genève : Droz.

Wilson, Deirdre (2006). «Pertinence et pragmatique lexicale ». Nouveaux cahiers de linguistique française 27, 33-52. 


\section{Textes cités}

le Roi, Adenet, Berte aus grans piés (après 1273-1274) ; texte cité d'après Littré (1961/62) et Detges (1999).

Le roman de Jehan de Paris (entre fin novembre 1494 et début décembre 1495), publié d'après les manuscrits par Edith Wickersheimer, Paris, Champion, Société des anciens textes, 1923 (repr. 1968) ; texte cité d'après Werner (1980).

Le roman de Renart (fin $12^{\mathrm{e}}$ - début $13^{\mathrm{e}}$ siècle), Méon, texte cité d'après Littré (1961/62) et Detges (1999).

Moralité de Charité (entre 1532 et 1550). In : Ancien théâtre françois, III, 338, éd. Viollet-le-Duc, Paris, 1855 ; texte cité d'après Gougenheim (1929/1971) et Detges (1999).

Rabelais, Pantagruel (1532). In : Les cinq livres, éd. Marty-Laveaux, Paris ; texte cité d'après Gougenheim (1929) et Detges (1999).

Racine, Jean. Andromaque (1667). In : CEuvres complètes, Paris, 1807 ; texte cité d'après Littré (1961/62) et Detges (1999).

\footnotetext{
${ }^{1}$ Pour une présentation des différentes approches et des références utiles, voir Traugott et Dasher (2002) et Hopper et Traugott (2003)

${ }^{2}$ On notera que Nicolle (1998) admet qu'une forme peut avoir son sens conceptuel originel (ou un sens conceptuel qui s'en est développé - il parle alors de semantic retention) et un sens procédural.

${ }^{3}$ Dans les termes de Ruiz de Mendoza Ibáñez et Hernández $(2003: 29)$ : « (...) the kind of metaphoric or metonymic connection is not a matter of the principle of relevance as such. What this principle does is merely draw our attention as interpreters to the necessity of making a connection. Understanding how this connection is made and finding out its communicative consequences depends on our ability to determine the cognitive operation to be carried out for the sentence to be relevant in the context. »
} 